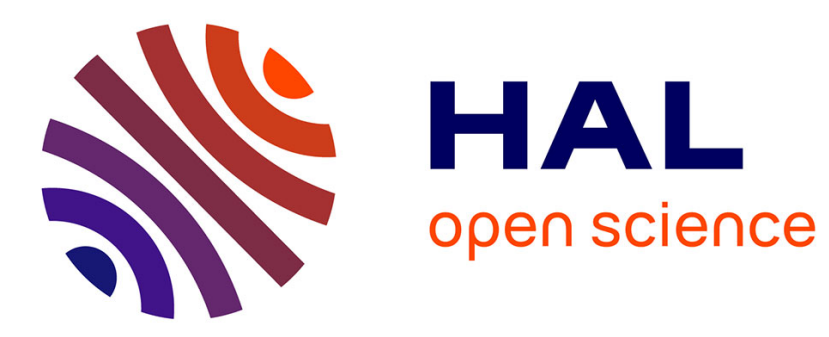

\title{
Is Randomness "Native" to Computer Science?
}

\author{
Marie Ferbus-Zanda, Serge Grigorieff
}

\section{To cite this version:}

Marie Ferbus-Zanda, Serge Grigorieff. Is Randomness "Native" to Computer Science?. G. Paun, G. Rozenberg, A. Salomaa. Current Trends in Theoretical Computer Science. Vol2, World Scientific, pp.141-180, 2004. hal-00201576

\section{HAL Id: hal-00201576 https://hal.science/hal-00201576}

Submitted on 31 Dec 2007

HAL is a multi-disciplinary open access archive for the deposit and dissemination of scientific research documents, whether they are published or not. The documents may come from teaching and research institutions in France or abroad, or from public or private research centers.
L'archive ouverte pluridisciplinaire HAL, est destinée au dépôt et à la diffusion de documents scientifiques de niveau recherche, publiés ou non, émanant des établissements d'enseignement et de recherche français ou étrangers, des laboratoires publics ou privés. 


\section{Is Randomness "native" to Computer Science?}

\author{
Marie Ferbus-Zanda * \\ ferbus@logique.jussieu.fr
}

\author{
Serge Grigorieff * \\ seg@liafa.jussieu.fr
}

August 2003

Original paper published in

Current Trends in Theoretical Computer Science.

G. Paun, G. Rozenberg, A. Salomaa (eds.).

World Scientific, Vol.2, p.141-180, 2004

Earlier version in

Yuri Gurevich's "Logic In Computer Science Column"

Bulletin of EATCS, vol. 74, p.78-118, June 2001

\section{Contents}

1 From probability theory to Kolmogorov complexity 3

1.1 Randomness and Probability theory ................ 3

1.2 Intuition of finite random strings and Berry's paradox . . . . . . . . . . . 5

1.3 Kolmogorov complexity relative to a function $\ldots \ldots \ldots \ldots$

8 Why binary programs? . . . . . . . . . . . . . . . . . . . . 8

1.5 What about other possible outputs? . . . . . . . . . . . 8

2 Optimal Kolmogorov complexity $\quad 8$

2.1 The Invariance Theorem $\ldots \ldots \ldots \ldots$. . . . . . . . . . . . . . . 8

2.2 Coding pairs of strings . . . . . . . . . . . . . . . . . . . . . . . . 11

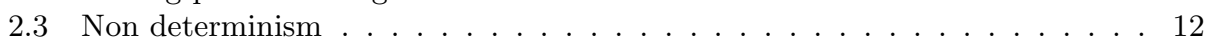

3 How complex is Kolmogorov complexity? 13

3.1 Approximation from abovt . . . . . . . . . . . . . . 13

3.2 Dovetailing . . . . . . . . . . . . . . . . . . . . . . . . . . . . . 14

3.3 Undecidability . . . . . . . . . . . . . . . . . . . . . . . . . . . . 15

3.4 No non trivial computable lower bound . . . . . . . . . . . . . . . . 16

3.5 Kolmogorov complexity and representation of objects . . . . . . . . . . . . 16

*LIAFA, CNRS \& Université Paris 7, 2, pl. Jussieu 75251 Paris Cedex 05 France 
4 Algorithmic Information Theory 17

4.1 Zip/Unzip . . . . . . . . . . . . . . . . . . . . . . . . . 17

4.2 Some relations in Algorithmic Information Theory . . . . . . . . . . . . . 18

4.3 Kolmogorov complexity of pairs . . . . . . . . . . . . . . . . . . . . 18

4.4 Symmetry of information $\ldots \ldots \ldots \ldots \ldots$

5 Kolmogorov complexity and Logic 19

5.1 What to do with paradoxes . . . . . . . . . . . . . . . . . . . . 19

5.2 Chaitin Incompleteness results . . . . . . . . . . . . . . . . . . . . . 20

5.3 Logical complexity of $K \ldots \ldots \ldots \ldots \ldots \ldots$

6 Random finite strings and their applications 22

6.1 Random versus how much random . . . . . . . . . . . . . . . . . . . 22

6.2 Applications of random finite strings in computer science . . . . . . . . . . 23

\begin{tabular}{lll}
\hline & Prefix complexity & 24
\end{tabular}

7.1 Self delimiting programs . . . . . . . . . . . . . . . . . . . 24

7.2 Chaitin-Levin prefix complexity . . . . . . . . . . . . . . . 25

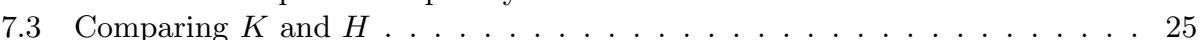

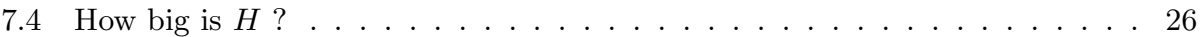

7.5 Convergence of series and the Coding Theorem . . . . . . . . . . . 26

8 Random infinite sequences $\quad 27$

8.1 Top-down approach to randomness of infinite sequences . . . . . . . . . 27

8.2 Frequency tests and von Mises random sequences . . . . . . . . . . . . . 28

8.3 Martin-Löf random sequences . . . . . . . . . . . . . . . . . . 29

8.4 Bottom-up approach to randomness of infinite sequences: Martin-Löf's

Large oscillations theorem $\ldots \ldots \ldots 32$

8.5 Bottom-up approach with prefix complexity . . . . . . . . . . . . . . . . 33

8.6 Top-down/Bottom-up approaches: a sum up . . . . . . . . . . . . . . . . 33

8.7 Randomness with other probability distributions . . . . . . . . . . . . . . . 34

8.8 Chaitin's real $\Omega \ldots \ldots \ldots \ldots \ldots$

8.9 Non computable invariance $\ldots \ldots \ldots \ldots$

$\begin{array}{llr}9 & \text { More randomness } & 36\end{array}$

9.1 Beyond c.e.: oracles and infinite computations . . . . . . . . . . . . . . . . 37

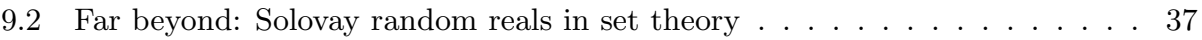




\section{From probability theory to Kolmogorov com- plexity}

\subsection{Randomness and Probability theory}

Quisani $^{1}$ : I just found a surprising assertion on Leonid Levin's home page:

While fundamental in many areas of Science, randomness is really "native" to Computer Science.

Common sense would rather consider randomness as intrinsically relevant to Probability theory!

Authors: Levin also adds: "The computational nature of randomness was clarified by Kolmogorov."

The point is that, from its very origin to modern axiomatization around 1933 [21] by Andrei Nikolaievitch Kolmogorov (1903-1987), Probability theory carries a paradoxical result:

if we toss an unbiaised coin 100 times then 100 heads are just as probable as any other outcome!

As Peter Gács pleasingly remarks ([17], p. 3), this convinces us only that the axioms of Probability theory, as developped in [21], do not solve all mysteries that they are sometimes supposed to.

In fact, since Laplace, much work has been devoted to get a mathematical theory of random objects, notably by Richard von Mises (1883-1953) (cf. $\S$.2.). But none was satisfactory up to the 60 's when such a theory emerged on the basis of computability.

As it sometimes occurs, the theory was discovered by several authors independently. ${ }^{2}$ In the USA, Ray J. Solomonoff (b. 1926), 1964 [42] (a paper submitted in 1962) and Gregory J. Chaitin (b. 1947), 1966 [5], 1969 [6] (both papers submitted in 1965). In Russia, Kolmogorov, 1965 [23], with premisses announced in 1963 [22].

Q: Same phenomenon as for hyperbolic geometry with Gauss, Lobatchevski and Bolyai. I recently read a citation from Bolyai's father: "When the time is ripe for certain things, these things appear in different places in the manner of violets coming to light in early spring".

\footnotetext{
${ }^{1}$ Quisani is a student with quite eclectic scientific curiosity, who works under Yuri Gurevich's supervision

${ }^{2}$ For a detailed analysis of who did what, and when, see [30] p.89-92.
} 
A: Mathematics and poetry... Well, pioneered by Kolmogorov, Martin-Löf, Levin, Gács, Schnorr (in Europe) and Chaitin, Solovay (in America), the theory developped very fruitfully and is now named Kolmogorov complexity or Algorithmic Information Theory.

Q: So, Kolmogorov founded Probability Theory twice! In the 30's and then in the 60 's.

A: Hum... In the 30's Kolmogorov axiomatized Probability Theory on the basis of measure theory, i.e. integration theory on abstract spaces. In the 60's, Kolmogorov (and also Solomonoff and Chaitin independently) founded a mathematical theory of randomness. That it could be a new basis for Probability Theory is not clear.

Q: What? Randomness would not be the natural basis for Probability Theory?

A: Random numbers are useful in different kinds of applications: simulations of natural phenomena, sampling for testing "typical case", getting good source of data for algorithms, ... (cf. Donald Knuth, [20], chapter 3).

However, the notion of random object as a mathematical notion is presently ignored in lectures about Probability Theory. Be it for the foundations or for the development of Probability Theory, such a notion is neither introduced nor used. That's the way it is. . There is a notion of random variable, but it has really nothing to do with random objects. Formally, they are just functions over some probability space. The name "random variable" is a mere vocable to convey the underlying non formalized intuition of randomness.

Q: That's right. I attended several courses on Probability Theory. Never heard anything precise about random objects. And, now that you tell me, I realize that there was something strange for me with random variables.

So, finally, our concrete experience of chance and randomness on which we build so much intuition is simply removed from the formalization of Probability Theory.

Hum... Somehow, it's as if the theory of computability and programming were omitting the notion of program, real programs.

By the way, isn' it the case? In recursion theory, programs are reduced to mere integers: Gödel numbers!

A: Sure, recursion theory illuminates but does not exhaust the subject of programming.

As concerns a new foundation of Probability Theory, it's already quite remarkable that Kolmogorov has looked at his own work on the subject with 
such a distance. So much as to come to a new theory: the mathematization of randomness. However, it seems (to us) that Kolmogorov has been ambiguous on the question of a new foundation. Indeed, in his first paper on the subject (1965, 23], p. 7), Kolmogorov briefly evoked that possibility :

...to consider the use of the [Algorithmic Information Theory] constructions in providing a new basis for Probability Theory.

However, later (1983, [25], p. 35-36), he separated both topics

"there is no need whatsoever to change the established construction of the mathematical probability theory on the basis of the general theory of measure. I am not enclined to attribute the significance of necessary foundations of probability theory to the investigations [about Kolmogorov complexity] that I am now going to survey. But they are most interesting in themselves.

though stressing the role of his new theory of random objects for mathematics as a whole (25], p. 39):

The concepts of information theory as applied to infinite sequences give rise to very interesting investigations, which, without being indispensable as a basis of probability theory, can acquire a certain value in the investigation of the algorithmic side of mathematics as a whole."

Q: All this is really exciting. Please, tell me about this approach to randomness.

\subsection{Intuition of finite random strings and Berry's paradox}

A: OK. We shall first consider finite strings.

If you don't mind, we can start with an approach which actually fails but conveys the basic intuitive idea of randomness. Well, just for a while, let's say that a finite string $u$ is random if there is no shorter way to describe $u$ but give the successive symbols which constitute $u$. Saying it otherwise, the shortest description of $u$ is $u$ itself, i.e. the very writing of the string $u$.

Q: Something to do with intensionality and extensionality?

A: You are completely right. Our tentative definition declares a finite string to be random just in case it does not carry any intensionality. So that there is no description of $u$ but the extensional one, which is $u$ itself. 
Q: But the notion of description is somewhat vague. Is it possible to be more precise about "description" and intensionality?

A: Diverse partial formalizations are possible. For instance within any particular logical first-order structure. But they are quite far from exhausting the intuitive notion of definability. In fact, the untamed intuitive notion leads to paradoxes, much as the intuition of truth.

Q: I presume you mean the liar paradox as concerns truth. As for definability, it should be Berry's paradox about

"the smallest integer not definable in less than eleven words"

and this integer is indeed defined by this very sentence containing only 10 words.

A: Yes, these ones precisely. By the way, this last paradox was first mentioned by Bertrand Russell, 1908 ([38], p.222 or 150) who credited G.G. Berry, an Oxford librarian, for the suggestion.

\subsection{Kolmogorov complexity relative to a function}

Q: And how can one get around such problems?

A: What Solomonoff, Kolmogorov and Chaitin did is a very ingenious move: instead of looking for a general notion of definability, they restricted it to computability. Of course, computability is a priori as much a vague and intuitive notion as is definability. But, as you know, since the thirties, there is a mathematization of the notion of computability.

Q: Thanks to Kurt, Alan and Alonzo. ${ }^{3}$

A: Hum... Well, with such a move, general definitions of a string $u$ are replaced by programs which compute $u$.

Q: Problem: we have to admit Church's thesis.

A: OK. In fact, even if Church's thesis were to break down, the theory of computable functions would still remain as elegant a theory as you learned from Yuri and other people. It would just be a formalization of a proper part of computability, as is the theory of primitive recursive functions or elementary functions. As concerns Kolmogorov theory, it would still hold and surely get extension to such a new context.

\footnotetext{
${ }^{3}$ Kurt Gödel (1906-1978), Alan Mathison Turing (1912-1954), Alonzo Church (19031995).
} 
Q: But where do the programs come from? Are you considering Turing machines or some programming language?

A: Any partial computable function $A:\{0,1\}^{*} \rightarrow\{0,1\}^{*}$ is considered as a programming language. The domain of $A$ is seen as a family of programs, the value $A(p)$ - if there is any - is the output of program $p$. As a whole, $A$ can be seen both as a language to write programs and as the associated operational semantics.

Now, Kolmogorov complexity relative to $A$ is the function $K_{A}:\{0,1\}^{*} \rightarrow \mathbb{N}$ which maps a string $x$ to the length of shortest programs which output $x$ :

Definition 1. $K_{A}(x)=\min \{|p|: A(p)=x\}$

(Convention: $\min (\emptyset)=+\infty$, so that $K_{A}(x)=+\infty$ if $x$ is outside the range of $A$ ).

Q: This definition reminds me of a discussion I had with Yuri some years ago (19] p.76-78). Yuri explained me things about Levin complexity. I remember it involved time.

A: Yes. Levin complexity is a very clever variant of $K$ which adds to the length of the program the log of the computation time to get the output. It's a much finer notion. We shall not consider it for our discussion about randomness. You'll find some developments in [30] $\S 7.5$.

Q: There are programs and outputs. Where are the inputs?

A: We can do without inputs. It's true that functions with no argument are not considered in mathematics, but in computer science, they are. In fact, since Von Neumann, we all know that there can be as much tradeof as desired between input and program. This is indeed the basic idea for universal machines and computers.

Nevertheless, Kolmogorov [23] points a natural role for inputs when considering conditional Kolmogorov complexity in a sense very much alike that of conditional probabilities.

To that purpose, consider a partial computable function $B:\{0,1\}^{*} \times$ $\{0,1\}^{*} \rightarrow\{0,1\}^{*}$. A pair $(p, y)$ in the domain of $B$ is interpreted as a program $p$ together with an input $y$. And $B(p, y)$ is the output of program $p$ on input $y$. Kolmogorov [23] defines the conditional complexity relative to $B$ as the function $K_{B}(\mid):\{0,1\}^{*} \times\{0,1\}^{*} \rightarrow \mathbb{N}$ which maps strings $x, y$ to the length of shortest programs which output $x$ on input $y$ :

Definition 2. $K_{B}(x \mid y)=\min \{|p|: B(p, y)=x\}$ 


\subsection{Why binary programs?}

Q: Programs should be binary strings?

A: This is merely a reasonable restriction.

Binary strings surely have some flavor of machine level programming. But this has nothing to do with the present choice. In fact, binary strings just allow for a fairness condition. The reason is that Kolmogorov complexity deals with lengthes of programs. Squaring or cubing the alphabet divides all lengthes by 2 or 3 as we see when going from binary to octal or hexadecimal. So that binary representation of programs is merely a way to get an absolute measure of length. If we were to consider programs $p$ written in some finite alphabet $\Sigma$, we would have to replace the length $|p|$ by the product $|p| \log (\operatorname{card}(\Sigma))$ where $\operatorname{card}(\Sigma)$ is the number of symbols in $\Sigma$. This is an important point when comparing Kolmogorov complexities associated to diverse programming languages, cf. 2.1.

\subsection{What about other possible outputs?}

Q: Outputs should also be binary strings?

A: Of course not. In Kolmogorov approach, outputs are the finite objects for which a notion of randomness is looked for. Binary strings constitute a simple instance. One can as well consider integers, or rationals or elements of any structure $D$ with a natural notion of computability. The modification is straightforward: now $A$ is a partial computable function $A:\{0,1\}^{*} \rightarrow D$ and $K_{A}: D \rightarrow \mathbb{N}$ is defined in the same way: $K_{A}(x)$, for $x \in D$, is the minimum length of a program $p$ such that $f(p)=x$.

\section{Optimal Kolmogorov complexity}

\subsection{The Invariance Theorem}

Q: Well, for each partial computable function $A:\{0,1\}^{*} \rightarrow\{0,1\}^{*}$ (or $A:\{0,1\}^{*} \rightarrow D$, as you just explained) there is an associated Kolmogorov complexity. So, what is the Kolmogorov complexity of a given string? It depends on the chosen $A$.

A: Now, comes the fundamental result of the theory, the so-called invariance theorem. We shall state it uniquely for Kolmogorov complexity but it also holds for conditional Kolmogorov complexity.

Recall the enumeration theorem: partial computable functions can be enumerated in a partial computable way. This means that there exists a partial 
computable function $E: \mathbb{N} \times\{0,1\}^{*} \rightarrow\{0,1\}^{*}$ such that, for every partial computable function $A:\{0,1\}^{*} \rightarrow\{0,1\}^{*}$, there is some $e \in \mathbb{N}$ for which we have $\forall p A(p)=E(e, p)$ (equality means that $A(p)$ and $E(e, p)$ are simultaneously defined or not and, if defined, of course they must be equal).

Q: Wait, wait, I remember the diagonal argument which proves that there is no enumeration of functions $\mathbb{N} \rightarrow \mathbb{N}$. It goes through computability. Given $E: \mathbb{N} \times\{0,1\}^{*} \rightarrow\{0,1\}^{*}$, the function $A: \mathbb{N} \rightarrow \mathbb{N}$ such that $A(n)=$ $E(n, n)+1$ is different from each one of the function $n \mapsto E(n, e)$ 's. And if $E$ is computable then $A$ is computable.

So, how can there be an enumeration of computable functions?

A: There is no computable enumeration of computable functions. The diagonal argument you recalled proves that this is impossible. No way.

But, we are not considering computable functions but partial computable functions. This makes a big difference. The diagonal argument breaks down. In fact, equality $E(n, n)=E(n, n)+1$ is not incoherent: it just insures that $E(n, n)$ is not defined!

Q: Very strange property,indeed.

A: No, no. It's quite intuitive nowadays, in our world with computers. Given a program in some fixed programming language, say language LISP, an interpreter executes it. Thus, with one more argument, the simulated program, the LISP compiler enumerates all functions which can be computed by a LISP program. Now, any partial computable function admits a LISP program. Thus, the LISP interpreter gives you a computable enumeration of computable functions.

Q: OK.

A: Let's go back to the invariance theorem. We transform $E$ into a one argument partial computable function $U:\{0,1\}^{*} \rightarrow\{0,1\}^{*}$ as follows: Set

$\left\{\begin{aligned} U\left(0^{e} 1 p\right) & =E(e, p) \\ U(q) & =\text { undefined if } q \text { contains no occurrence of } 1\end{aligned}\right.$

(where $0^{e}$ is the string $00 \ldots 0$ with length $e$ ).

Then, if $A:\{0,1\}^{*} \rightarrow\{0,1\}^{*}$ is partial computable and $e_{0}$ is such that $\forall p A(p)=E\left(e_{0}, p\right)$, we have 


$$
\begin{array}{rlrl}
K_{U}(x) & =\min \{|q|: U(q)=x\} & & \text { (definition of } \left.K_{U}\right) \\
& =\min \left\{\left|0^{e} 1 p\right|: U\left(0^{e} 1 p\right)=x\right\} & \\
& \leq \min \left\{\left|0^{e_{0}} 1 p\right|: U\left(0^{e} 1 p\right)=x\right\} & & \left(\text { restriction to } e=e_{0}\right) \\
& =\min \left\{\left|0^{e_{0}} 1 p\right|: A(p)=x\right\} & & (e \text { is a code for } A) \\
& =e+1+\min \{|p|: A(p)=x\} & & \\
& =e+1+K_{A}(x) & & \left(\text { definition of } K_{A}\right)
\end{array}
$$

Let's introduce useful notations. For $f, g:\{0,1\}^{*} \rightarrow \mathbb{N}$, let's write

$$
f \leq g+O(1) \quad(\text { resp. } f=g+O(1))
$$

to mean that there exists a constant $\mathrm{c}$ such that

$$
\forall x f(x) \leq g(x)+c \quad(\text { resp. } \forall x|f(x)-g(x)| \leq c)
$$

i.e. $f$ is smaller than (resp. equal to) $g$ up to an additive constant $c$.

What we have just shown can be expressed as the following theorem independently obtained by Kolmogorov (1965 [23] p.5), Chaitin (1966 [5] §9-11) and Solomonoff (1964 [42] p.12, who gives the proof as an informal argument).

Theorem 3 (Invariance theorem). $K_{U} \leq K_{A}+O(1)$ for any partial computable $A:\{0,1\}^{*} \rightarrow\{0,1\}^{*}$. In other words, up to an additive constant, $K_{U}$ is the smallest one among the $K_{A}$ 's.

Thus, up to an additive constant, there is a smallest $K_{A}$. Of course, if $K_{U}$ and $K_{V}$ are both smallest, up to an additive constant, then $K_{U}=K_{V}+O(1)$. Whence the following definition.

Definition 4 (Kolmogorov complexity). Kolmogorov complexity $K$ : $\{0,1\}^{*} \rightarrow \mathbb{N}$ is any fixed such smallest ( $\mathrm{p}$ to an additive constant) function $K_{U}$.

Let's sum up. The invariance theorem means that, up to an additive constant, there is an intrinsic notion of Kolmogorov complexity and we can speak of the Kolmogorov complexity of a binary string.

Q: Which is an integer defined up to a constant... Somewhat funny.

A: You witty! Statements that only make sense in the limit occur everywhere in mathematical contexts.

Q: Do not mind, I was joking.

A: In fact, Kolmogorov argued as follows about the constant, 23] p. 6: 
Of course, one can avoid the indeterminacies associated with the [above] constants, by considering particular [...functions U], but it is doubtful that this can be done without explicit arbitrariness. One must, however, suppose that the different "reasonable" [above universal functions] will lead to "complexity estimates" that will converge on hundreds of bits instead of tens of thousands. Hence, such quantities as the "complexity" of the text of "War and Peace" can be assumed to be defined with what amounts to uniqueness.

Q: Using the interpretation you mentioned a minute ago with programming languages concerning the enumeration theorem, the constant in the invariance theorem can be viewed as the length of a LISP program which interprets $A$.

A: You are right.

\subsection{Coding pairs of strings}

A: Have you noted the trick to encode an integer $e$ and a string $p$ into a string $0^{e} 1 p$ ?

Q: Yes, and the constant is the length of the extra part $0^{e} 1$. But you have encoded $e$ in unary. Why not use binary representation and thus lower the constant?

A: There is a problem. It is not trivial to encode two binary strings $u, v$ as a binary string $w$. We need a trick. But first, let's be clear: encode here means to apply a computable injective function $\{0,1\}^{*} \times\{0,1\}^{*} \rightarrow\{0,1\}^{*}$. Observe that concatenation does not work: if $w=u v$ then we don't know which prefix of $w$ is $u$. A new symbol 2 inserted as a marker allows for an encoding: from $w=u 2 v$ we can indeed recover $u, v$. However, $w$ is no more a binary string.

A simple solution uses this last idea together with a padding function applied to $u$ which allows 1 to become an end-marker. Let $\operatorname{pad}(u)$ be obtained by inserting a new zero in front of every symbol in $u$. For instance, $\operatorname{pad}(01011)=0001000101$. Now, a simple encoding $w$ of strings $u, v$ is the concatenation $w=\operatorname{pad}(u) 1 v$. In fact, the very definition of pad insures that the end of the prefix $\operatorname{pad}(u)$ in $w$ is marked by the first occurrence of 1 at an odd position (obvious convention: first symbol has position 1). Thus, from $w$ we get $\operatorname{pad}(u)$ — hence $u$ - and $v$ in a very simple way: a finite 
automaton can do the job! Observe that

$$
|\operatorname{pad}(u) 1 v|=2|u|+|v|+1
$$

Q: Is the constant 2 the best one can do?

A: No, one can iterate the trick. Instead of padding $u$, one can pad the string $\overline{|u|}$ which is the binary representation of the length of $u$. Look at the string $w=\operatorname{pad}(\overline{|u|}) 1 u v$. The first occurrence of 1 at an odd position tells you which prefix of $w$ is $\operatorname{pad}(\overline{|u|})$ and which suffix is $u v$. From the prefix $\operatorname{pad}(\overline{|u|})$, you get $\overline{|u|}$, hence $|u|$. From $|u|$ and the suffix $u v$, you get $u$ and $v$. Nice trick, isn't it? And since $|\overline{\mid u \|}|=1+\lfloor\log (|u|)\rfloor$, we get

$$
|\operatorname{pad}(\overline{|u|}) 1 u v|=|u|+|v|+2\lfloor\log (|u|)\rfloor+3
$$

Q: Exciting! One could altogether pad the length of the length.

A: Sure. $\operatorname{pad}(\overline{\mid \overline{|u|}}) 1 \overline{|u|} u v$ is indeed an encoding of $u, v$. The first occurrence of 1 at an odd position tells you which prefix of $w$ is $\operatorname{pad}(\overline{\mid \overline{|u|}})$ and which suffix is $\overline{|u|} u v$. From the prefix pad( $(\overline{\mid \overline{|u|}})$, you get $\overline{\mid \overline{|u|}}$ hence $|\overline{\mid u \|}|$. Now, from $|\overline{|u|}|$ and the suffix $\overline{|u|} u v$ you get $\overline{|u|}$ - hence $|u|-$ and $u v$. From $|u|$ and $u v$, you get $u$ and $v$. Also, a simple computation leads to

$$
|\operatorname{pad}(\overline{\mid \overline{|u|}}) 1 \overline{|u|} u v|=|u|+|v|+\lfloor\log (|u|)\rfloor+2\lfloor\log (1+\lfloor\log (|u|)\rfloor)\rfloor+3
$$

Q: Of course, we can iterate this process.

A: Right. But, let's leave such refinements.

\subsection{Non determinism}

Q: Our problematic is about randomness. Chance, randomness, arbitrariness, unreasonned choice, non determinism ... Why not add randomness to programs by making them non deterministic with several possible outputs?

A: Caution: if a single program can output every string then Kolmogorov complexity collapses. In order to get a non trivial theory, you need to restrict non determinism. There is a lot of reasonable ways to do so. It happens that all lead to something which is essentially usual Kolmogorov complexity up to some change of scale (41], [18]). Same with the prefix Kolmogorov complexity which we shall discuss later. 


\section{How complex is Kolmogorov complexity?}

Q: Well, let me tell you some points I see about $K_{A}$.

The domain of $K_{A}$ appears to be the range of $A$. So that $K_{A}$ is total in case $A$ is onto.

Since there are finitely many programs $p$ with length $\leq n$, there can be only finitely many $x$ 's such that $K_{A}(x) \leq n$. So that, $\lim _{|x| \rightarrow+\infty} K_{A}(x)=+\infty$.

Also, in the definition of $K_{A}(x)$, there are 2 points:

1) find some program which outputs $x$,

2) make sure that all programs with shorter length either do not halt or have output different from $x$.

Point 2 does not match with definitions of partial computable functions!

\subsection{Approximation from above}

A: Right. In general, $K_{A}$ is not partial computable.

Q: So, no way to compute $K_{A}$.

A: Definitely not, in general. However, $K_{A}$ can be approximated from above:

Proposition 5. $K_{A}$ is the limit of a computable decreasing sequence of functions.

Moreover, we can take such a sequence of functions with finite domains.

To see this, fix an algorithm $\mathcal{A}$ for $A$ and denote $A_{t}$ the partial function obtained by applying up to $t$ steps of algorithm $\mathcal{A}$ for the sole programs with length $\leq t$. It is clear that $(t, p) \mapsto A_{t}(p)$ has computable graph. Also,

$$
K_{A_{t}}(x)=\min \left\{|p|: p \in\{0,1\}^{\leq t} \text { and } A_{t}(p)=x\right\}
$$

so that $(t, x) \mapsto K_{A_{t}}(x)$ has computable graph too.

To conclude, just observe that $(t, x) \mapsto K_{A_{t}}(x)$ is decreasing in $t$ (with the obvious convention that undefined $=+\infty)$ and that $K_{A}(x)=\lim _{t \rightarrow \infty} K_{A_{t}}(x)$. The same is true for conditional Kolmogorov complexity $K_{B}(\mid)$.

Q: If $K_{A}$ is not computable, there should be no computable modulus of convergence for this approximation sequence. So what can it be good for?

A: In general, if a function $f:\{0,1\}^{*} \rightarrow \mathbb{N}$ can be approximated from above by a computable sequence of functions $\left(f_{t}\right)_{t \in \mathbb{N}}$ then $X_{n}^{f}=\{x: f(x) \leq n\}$ is computably enumerable (in fact, both properties are equivalent). Which is a very useful property of $f$. Indeed, such arguments are used in the proof of some hard theorems in the subject of Kolmogorov complexity. 
Q: Could you give me the flavor of what it can be useful for?

A: Suppose you know that $X_{n}^{f}$ is finite (which is indeed the case for $f=K_{A}$ ) and has exactly $m$ elements then you can explicitly get $X_{n}^{f}$.

Q: Explicitly get a finite set? what do you mean?

A: What we mean is that there is a computable function which associates to any $m, n$ a code (in whatever modelization of computability) for a partial computable function which has range $X_{n}^{f}$ in case $m$ is equal to the number of elements in $X_{n}^{f}$. This is not trivial. We do this thanks to the $f_{t}$ 's.

Indeed, compute the $f_{t}(x)$ 's for all $t$ 's and all $x$ 's until you get $m$ different strings $x_{1}, \ldots, x_{m}$ such that $f_{t_{1}}\left(x_{1}\right), \ldots, f_{t_{m}}\left(x_{m}\right)$ are defined and $\leq n$ for some $t_{1}, \ldots, t_{m}$.

That you will get such $x_{1}, \ldots, x_{m}$ is insured by the fact that $X_{n}^{f}$ has at least $m$ elements and that $f(x)=\min \left\{f_{t}(x): t\right\}$ for all $x$.

Since $f \leq f_{t}$, surely these $x_{i}$ 's are in $X_{n}^{f}$. Moreover, they indeed constitute the whole of $X_{n}^{f}$ since $X_{n}^{f}$ has exactly $m$ elements.

\subsection{Dovetailing}

Q: You run infinitely many computations, some of which never halt. How do you manage them?

A: This is called dovetailing. You organize these computations (which are infinitely many, some lasting forever) as follows:

- Do up to 1 computation step of $f_{t}(x)$ for $0 \leq t \leq 1$ and $0 \leq|x| \leq 1$,

- Do up to 2 computation steps of $f_{t}(x)$ for $0 \leq t \leq 2$ and $0 \leq|x| \leq 2$,

- Do up to 3 computation steps of $f_{t}(x)$ for $0 \leq t \leq 3$ and $0 \leq|x| \leq 3$,

‥

Q: Somehow looks like Cantor's enumeration of $\mathbb{N}^{2}$ as the sequence
$(0,0)$
$(0,1)(1,0)$
$(0,2)(1,1)(2,0)$
$(0,3)(1,2)(2,1)(3,0) \ldots$

A: This is really the same idea. Here, it would rather be an enumeration à la Cantor of $\mathbb{N}^{3}$. When dealing with a multi-indexed family of computations $\left(\varphi_{t}(\vec{x})\right)_{\vec{t}}$, you can imagine computation steps as tuples of integers $(i, \vec{t}, \vec{x})$ where $i$ denotes the rank of some computation step of $f_{\vec{t}}(\vec{x})$ (here, these tuples are triples). Dovetailing is just a way of enumerating all points in a discrete multidimensional space $\mathbb{N}^{k}$ via some zigzagging à la Cantor.

Q: Well, Cantor wandering along a broken line which fills the discrete plane here becomes a way to sequentialize parallel computations. 


\subsection{Undecidability}

Q: Let's go back to $K_{A}$. If $A$ is onto then $K_{A}$ is total. So that if $A$ is also computable then $K_{A}$ should be computable too. In fact, to get $K_{A}(x)$ just compute all $A(p)$ 's, for increasing $|p|$ 's until some has value $x$ : this will happen since $A$ is onto.

You said $K_{A}$ is in general undecidable. Is this undecidability related to the fact that $A$ be partial computable and not computable?

A: No. It's possible that $K_{A}$ be quite trivial with $A$ as complex as you want. Let $f:\{0,1\}^{*} \rightarrow \mathbb{N}$ be any partial computable function. Set $A(0 x)=x$ and $A\left(1^{1+f(x)} 0 x\right)=x$. Then, $A$ is as complex as $f$ though $K_{A}$ is trivial since $K_{A}(x)=|x|+1$, as is easy to check.

Q: Is it hard to prove that some $K_{A}$ is indeed not computable?

A: Not that much. If $U:\{0,1\}^{*} \rightarrow\{0,1\}^{*}$ is optimal then we can show that $K_{U}$ is not computable. Thus, $K$ (which is $K_{U}$ for some fixed optimal $U)$ is not computable.

And this is where Berry's paradox comes back. Consider the length-lexicographic order on binary strings: $u<_{h i e r} v$ if and only if $|u|<|v|$ or $|u|=|v|$ and $u$ is lexicographically before $v$.

Now, look, we come to the core of the argument. The key idea is to introduce the function $T: \mathbb{N} \rightarrow\{0,1\}^{*}$ defined as follows:

$$
T(i)=\text { the }<_{\text {hier }} \text { smallest } x \text { such that } K_{U}(x)>i
$$

As you see, this function is nothing but an implementation of the very statement in Berry's paradox modified according to Kolmogorov's move from definability to computability via the function $A$. Clearly, we have

$$
K_{U}(T(i))>i
$$

Suppose, by way of contradiction, that $K_{U}$ is computable. Then so is $T$ and so is the function $V:\{0,1\}^{*} \rightarrow\{0,1\}^{*}$ such that $V(p)=T\left(\operatorname{Val}_{2}(1 p)\right)$ where $\operatorname{Val}_{2}(1 p)$ is the integer with binary representation $1 p$.

Now, if $i>0$ has binary representation $1 p$ then $T(i)=V(p)$, so that

$$
K_{V}(T(i)) \leq|p|=\lfloor\log (i)\rfloor
$$

The invariance theorem insures that, for some $c$, we have

$$
K_{U} \leq K_{V}+c
$$


From inequalities (4), (5), (6) we get

$$
i<K_{U}(T(i)) \leq K_{V}(T(i))+c \leq \log (i)+c
$$

which is a contradiction for $i$ large enough since $\lim _{i \rightarrow+\infty} \frac{\log (i)}{i}=0$. Thus, our assumption that $K_{U}$ be computable is false.

\subsection{No non trivial computable lower bound}

Q: Quite nice an argument.

A: Can get much more out of it:

Theorem 6. 1) No restriction of $K_{U}$ to an infinite computable set is computable.

2) Worse, if $X \subseteq\{0,1\}^{*}$ is computable and $f: X \rightarrow \mathbb{N}$ is a computable function and $f(x) \leq K_{U}(x)$ for all $x \in X$ then $f$ is bounded!

To prove this, just change the above definition of $T: \mathbb{N} \rightarrow\{0,1\}^{*}$ as follows:

$$
T(i)=\text { the }<_{\text {hier }} \text { smallest } x \in X \text { such that } f(x)>i
$$

Clearly, by definition, we have $f(T(i))>i$. Since $T(i) \in X$ and $f(x) \leq$ $K_{U}(x)$ for $x \in X$, this implies equation (四) above. Also, $f$ being computable, so are $T$ and $V$, and equation (5) still holds. As above, we conclude to a contradiction.

Let's reformulate this result in terms of the greatest monotonous (with respect to $\leq_{h i e r}$ ) lower bound of $K_{U}$ which is

$$
m(x)=\min _{y \geq \text { hier }} K_{U}(y)
$$

This function $m$ is monotonous and tends to $+\infty$ but it does so incredibly slowly: on any computable set it can not grow as fast as any unbounded computable function.

\subsection{Kolmogorov complexity and representation of objects}

Q: You have considered integers and their base 2 representations. Complexity of algorithms is often much dependent on the way objects are represented. Here, you have not be very precise about representation of integers.

A: There is a simple fact.

Proposition 7. Let $f:\{0,1\}^{*} \rightarrow\{0,1\}^{*}$ be partial computable.

1) $K(f(x)) \leq K(x)+O(1)$ for every $x$ in the domain of $f$.

2) If $f$ is also injective then $K(f(x))=K(x)+O(1)$ for $x \in$ domain $(f)$. 
Indeed, denote $U$ some fixed universal function such that $K=K_{U}$.

To get a program which outputs $f(x)$, we just encode a program $\pi$ computing $f$ together with a program $p$ outputting $x$.

Formally, let $A:\{0,1\}^{*} \rightarrow\{0,1\}^{*}$ be such that $A(\operatorname{pad}(\pi) 1 z)$ is the output of $\pi$ on input $U(z)$ for all $z \in\{0,1\}^{*}$. Clearly, $A(\operatorname{pad}(\pi) 1 p)=f(x)$ so that $K_{A}(f(x)) \leq 2|\pi|+|p|+1$. Taking $p$ such that $K(x)=|p|$, we get $K_{A}(f(x)) \leq K(x)+2|\pi|+1$.

The Invariance Theorem insures that $K(f(x)) \leq K_{A}(f(x))+O(1)$, whence point 1 of the Proposition.

In case $f$ is injective, it has a partial computable inverse $g$ with domain the range of $f$. Applying point 1 to $f$ and $g$ we get point 2 .

A: So all representations of integers lead to the same Kolmogorov complexity, up to a constant.

A: Yes, as long as one can computably go from one representation to the other one.

\section{Algorithmic Information Theory}

\subsection{Zip/Unzip}

Q: A moment ago, you said the subject was also named Algorithmic Information Theory. Why?

A: Well, you can look at $K(x)$ as a measure of the information contents that $x$ conveys. The notion can also be vividly described using our everyday use of compression/decompression software (cf. Alexander Shen's lecture 401). First, notice the following simple fact:

Proposition 8. $K(x) \leq|x|+O(1)$

Indeed, let $A(x)=x$. Then $K_{A}(x)=|x|$ and the above inequality is a mere application of the Invariance Theorem.

Looking at the string $x$ as a file, any program $p$ such that $U(p)=x$ can be seen as a compressed file for $x$ (especially in case the right member in Proposition 8) is indeed $<|x| \ldots$ ).

So, $U$ appears as a decompression algorithm which maps the compressed file $p$ onto the original file $x$. In this way, $K(x)$ measures the length of the shortest compressed files for $x$.

What does compression? It eliminates redundancies, explicits regularities to shorten the file. Thus, maximum compression reduces the file to the core of its information contents which is therefore measured by $K(x)$. 


\subsection{Some relations in Algorithmic Information Theory}

Q: OK. And what does Algorithmic Information Theory look like?

Q: Conditional complexity should give some nice relations as is the case with conditional probability.

A: Yes, there are relations which have some probability theory flavor. However, there are often logarithmic extra terms which come from the encoding of pairs of strings. For instance, an easy relation:

$$
K(x) \leq K(x \mid y)+K(y)+2 \log (\min (K(x \mid y), K(y)))+O(1)
$$

The idea to get this relation is as follows. Suppose you have a program $p$ (with no parameter) which outputs $y$ and a program $q$ (with one parameter) which on input $y$ outputs $x$, then you can mix them to get a (no parameter) program which outputs $x$.

Formally, Suppose that $p, q$ are optimal, i.e. $K(y)=|p|$ and $K(x \mid y)=|q|$. Let $A_{1}, A_{2}:\{0,1\}^{*} \rightarrow\{0,1\}^{*}$ be such that

$$
A_{1}(\operatorname{pad}(|z|) 1 z w)=A_{2}(\operatorname{pad}(|w|) 1 z w)=V(w, U(z))
$$

where $V$ denotes some fixed universal function such that $K(\mid)=K_{V}(\mid)$. It is clear that $A_{1}(\operatorname{pad}(|p|) 1 p q)=A_{2}(\operatorname{pad}(|q|) 1 p q)=x$, so that

$$
\begin{aligned}
& K_{A_{1}}(x) \leq|p|+|q|+2 \log (|p|)+O(1) \\
& K_{A_{2}}(x) \leq|p|+|q|+2 \log (|q|)+O(1)
\end{aligned}
$$

whence, $p, q$ being optimal programs,

$$
\begin{gathered}
K_{A_{1}}(x) \leq K(y)+K(x \mid y)+2 \log (K(y))+O(1) \\
K_{A_{2}}(x) \leq K(y)+K(x \mid y)+2 \log (K(x \mid y))+O(1)
\end{gathered}
$$

Applying the Invariance Theorem, we get (7).

\subsection{Kolmogorov complexity of pairs}

Q: What about pairs of strings in the vein of the probability of a pair of events?

A: First, we have to define the Kolmogorov complexity of pairs of strings. The key fact is as follows:

Proposition 9. If $f, g:\{0,1\}^{*} \times\{0,1\}^{*} \rightarrow\{0,1\}^{*}$ are encodings of pairs of strings (i.e. computable injections), then $K(f(x, y))=K(g(x, y))+O(1)$.

As we always argue up to an additive constant, this leads to:

Definition 10. The Kolmogorov complexity of pairs is $K(x, y)=K(f(x, y))$ where $f$ is any fixed encoding. 
To prove Proposition 9, observe that $f \circ g^{-1}$ is a partial computable injection such that $f=\left(f \circ g^{-1}\right) \circ g$. Then, apply Proposition 6 with argument $g(x, y)$ and function $f \circ g^{-1}$.

\subsection{Symmetry of information}

A: Relation (7) can be easily improved to

$$
K(x, y) \leq K(x \mid y)+K(y)+2 \log (\min (K(x \mid y), K(y)))+O(1)
$$

The same proof works. Just observe that from both programs $p a d(|p|) 1 p q$ and $\operatorname{pad}(|q|) 1 p q$ one gets $q$ hence also $y$.

Now, (8) can be considerably improved:

Theorem 11. $|K(x, y)-K(x \mid y)-K(y)|=O(\log (K(x, y))$

This is a hard result, independently obtained by Kolmogorov and Levin around 1967 (24], [50] p.117). We better skip the proof (you can get it in [40] p. 6-7 or [30] Thm 2.8.2 p. 182-183).

Q: I don't really see the meaning of that theorem.

A: Let's restate it in another form.

Definition 12. $I(x: y)=K(y)-K(y \mid x)$ is called the algorithmic information about $y$ contained in $x$.

This notion is quite intuitive: you take the difference between the whole information contents of $y$ and that when $x$ is known for free.

Contrarily to what was expected in analogy with Shannon's classical information theory, this is not a symmetric function However, up to a logarithmic term, it is symmetric:

Corollary 13. $|I(x: y)-I(y: x)|=O(\log (K(x, y))$

For a proof, just apply Theorem 11 with $K(x, y)$ and $K(y, x)$ and observe that $K(x, y)=K(y, x)+O(1)$ (use Proposition 9).

\section{Kolmogorov complexity and Logic}

\subsection{What to do with paradoxes}

Q: Somehow, Solomonoff, Kolmogorov and Chaitin have built up a theory from a paradox. 
A: Right. In fact, there seems to be two mathematical ways towards paradoxes. The most natural one is to get rid of them by building secured and delimited mathematical frameworks which will leave them all out (at least, we hope so... ). Historically, this was the way followed in all sciences. A second way, which came up in the 20th century, somehow integrates paradoxes into scientific theories via some clever and sound (!) use of the ideas they convey. Kolmogorov complexity is such a remarkable integration of Berry's paradox into mathematics.

Q: As Gödel did with the liar paradox which is underlying his incompleteness theorems. Can we compare these paradoxes?

A: Hard question. The liar paradox is about truth while Berry's is about definability. Viewed in computational terms, truth and definability somehow correspond to denotational and operational semantics.

This leads to expect connections between incompleteness theorems à la Gödel and Kolmogorov investigations.

\subsection{Chaitin Incompleteness results}

Q: So, incompleteness theorems can be obtained from Kolmogorov theory?

A: Yes. Gregory Chaitin, 1971 []], pointed and popularized a simple but clever and spectacular application of Kolmogorov complexity (this original paper by Chaitin did not consider $K$ but the number of states of Turing machines, which is much similar).

Let $\mathcal{T}$ be a computable theory containing Peano arithmetic such that all axioms of $\mathcal{T}$ are true statements.

Theorem 14. There exists a constant $c$ such that if $\mathcal{T}$ proves $K(x) \geq n$ then $n \leq c$.

The proof is by way of contradiction and is a redo of the undecidability of $K_{U_{P A S C A L^{b i n}}}$. Suppose that $\mathcal{T}$ can prove statements $K(x) \geq n$ for arbitrarily large $n$ 's. Consider a computable enumeration of all theorems of $\mathcal{T}$ and let $f: \mathbb{N} \rightarrow \mathbb{N}$ be such that $f(n)$ is the first string $x$ such that $K(x) \geq n$ appears as a theorem of $\mathcal{T}$. Our hypothesis insures that $f$ is total, hence a computable function. By very definition,

$$
K(\overline{f(n)} \geq n
$$

Also, applying Propositions 07 and 8 we get

$$
K(\overline{f(n)}) \leq K(\bar{n})+O(1) \leq \log (n)+O(1)
$$


whence $n \leq \log (n)+O(1)$, which is a contradiction if $n$ is large enough.

Q: Quite nice. But this does not give any explicit statement. How to compute the constant $c$ ? How to get any explicit $x$ 's such that $K(x)>c$ ?

A: Right. Hum... you could also see this as a particularly strong form of incompleteness: you have a very simple infinite family of statements, only finitely many can be proved but you don't know which ones.

\subsection{Logical complexity of $K$}

A: By the way, there is a point we should mention as concerns the logical complexity of Kolmogorov complexity.

Since $K$ is total and not computable, its graph can not be computably enumerable (c.e.). However, the graph of any $K_{A}$ (hence that of $K$ ) is always of the form $R \cap S$ where $R$ is c.e. and $S$ is co-c.e. (i.e. the complement of an c.e. relation).

We can see this as follows. Fix an algorithm $\mathrm{P}$ for $A$ and denote $A^{t}$ the partial function obtained by applying up to $t$ computation steps of this algorithm. Then

$$
K_{A}(x) \leq n \Leftrightarrow \exists t\left(\exists p \in\{0,1\}^{\leq n} A^{t}(p)=x\right)
$$

The relation within parentheses is computable in $t, n, x$, so that $K_{A}(x) \leq n$ is c.e. in $n, x$.

Replacing $n$ by $n-1$ and going to negations, we see that $K_{A}(x) \geq n$ is co-c.e. Since $K_{A}(x)=n \Leftrightarrow\left(K_{A}(x) \leq n\right) \wedge\left(K_{A}(x) \geq n\right)$, we conclude that $K_{A}(x)=n$ is the intersection of an c.e. and a co-c.e relations.

In terms of Post's hierarchy, the graph of $K_{A}$ is $\Sigma_{1}^{0} \wedge \Pi_{1}^{0}$, hence $\Delta_{2}^{0}$. The same with $K_{B}(\mid)$.

Q: Would you remind me about Post's hierarchy?

A: Emil Post introduced families of relations $R\left(x_{1} \ldots x_{m}\right)$ on strings and/or integers. Let's look at the first two levels:

$\Sigma_{1}^{0}$ and $\Pi_{1}^{0}$ are the respective families of c.e. and co-c.e. relations,

$\Sigma_{2}^{0}$ is the family of projections of $\Pi_{1}^{0}$ relations,

$\Pi_{2}^{0}$ consist of complements of $\Sigma_{2}^{0}$ relations.

Notations $\Sigma_{i}^{0}$ and $\Pi_{i}^{0}$ come from the following logical characterizations:

$R(\vec{x})$ is $\Sigma_{1}^{0}$ if $R(\vec{x}) \Leftrightarrow \exists t_{1} \ldots \exists t_{k} T(\vec{t}, \vec{x})$ with $T$ computable.

$R(\vec{x})$ is $\Sigma_{2}^{0}$ if $R(\vec{x}) \Leftrightarrow \exists \vec{t} \forall \vec{u} T(\vec{t}, \vec{u}, \vec{x})$ with $T$ computable.

$\Pi_{1}^{0}$ and $\Pi_{2}^{0}$ are defined similarly with quantifications $\forall$ and $\forall \exists$.

Each of these families is closed under union and intersection. But not under complementation since $\Sigma_{i}^{0}$ and $\Pi_{i}^{0}$ are so exchanged. 
A last notation: $\Delta_{i}^{0}$ denotes $\Sigma_{i}^{0} \cap \Pi_{i}^{0}$. In particular, $\Delta_{1}^{0}$ means c.e. and co-c.e. hence computable.

As for inclusion, $\Delta_{2}^{0}$ strictly contains the boolean closure of $\Sigma_{1}^{0}$, in particular it contains $\Sigma_{1}^{0} \cup \Pi_{1}^{0}$. This is why the term hierarchy is used.

Also, we see that $K_{A}$ is quite low as a $\Delta_{2}^{0}$ relation since $\Sigma_{1}^{0} \wedge \Pi_{1}^{0}$ is the very first level of the boolean closure of $\Sigma_{1}^{0}$.

\section{Random finite strings and their applications}

\subsection{Random versus how much random}

Q: Let's go back to the question: "what is a random string?"

A: This is the interesting question, but this will not be the one we shall answer. We shall modestly consider the question: "To what extent is $x$ random?"

We know that $K(x) \leq|x|+O(1)$. It is tempting to declare a string $x$ random if $K(x) \geq|x|-O(1)$. But what does it really mean? The $O(1)$ hides a constant. Let's explicit it.

Definition 15. A string is called $c$-incompressible (where $c \geq 0$ is any constant) if $K(x) \geq|x|-c$. Other strings are called $c$-compressible.

0 -incompressible strings are also called incompressible.

Q: Are there many $c$-incompressible strings?

A: Kolmogorov noticed that they are quite numerous.

Theorem 16. For each $n$ the proportion of c-incompressible among strings with length $n$ is $>1-2^{-c}$.

For instance, if $c=4$ then, for any length $n$, more than $90 \%$ of strings are 4-incompressible. With $c=7$ and $c=10$ we go to more than $99 \%$ and $99.9 \%$.

The proof is a simple counting argument. There are $1+2+2^{2}+\cdots+2^{n-c-1}=$ $2^{n-c}-1$ programs with length $<n-c$. Every string with length $n$ which is $c$-compressible is necessarily the output of such a program (but some of these programs may not halt or may output a string with length $\neq n$ ). Thus, there are at most $2^{n-c}-1 c$-compressible strings with length $n$, hence at least $2^{n}-\left(2^{n-c}-1\right)=2^{n}-2^{n-c}+1 c$-incompressible strings with length $n$. Whence the proportion stated in the theorem. 
Q: Are $c$-incompressible strings really random?

A: Yes. Martin-Löf, 1965 [34, formalized the notion of statistical test and proved that incompressible strings pass all these tests (cf. $\$ 8.3$ ).

\subsection{Applications of random finite strings in computer science}

Q: And what is the use of incompressible strings in computer science?

A: Roughly speaking, incompressible strings are strings without any form of local or global regularity. Consideration of such objects may help almost anytime one has to show something is complex, for instance a lower bound for worst or average case time/space complexity. The accompanying key tool is Proposition 0 .

And, indeed, incompressible strings have been successfully used in such contexts. An impressive compilation of such applications can be found in Ming Li and Paul Vitanyi's book ([30], chapter 6), running through nearly 100 pages!

Q: Could you give an example?

A: Sure. The very first such application is quite representative. It is due to Wolfgang Paul, 1979 [37] and gives a quadratic lower bound on the computation time of any one-tape Turing machine $\mathcal{M}$ which recognizes palindromes.

Up to a linear waste of time, one can suppose that $\mathcal{M}$ always halts on its first cell.

Let $n$ be even and $x x^{R}=x_{1} x_{2} \ldots x_{n-1} x_{n} x_{n} x_{n-1} \ldots x_{2} x_{1}$ be a palindrome written on the input tape of the Turing machine $\mathcal{M}$.

For each $i<n$ let $C S_{i}$ be the crossing sequence associated to cell $i$, i.e. the list of successive states of $\mathcal{M}$ when its head visits cell $i$.

Key fact: string $x_{1} x_{2} \ldots x_{i}$ is uniquely determined by $C S_{i}$.

I.e. $x_{1} x_{2} \ldots x_{i}$ is the sole string $y$ such that - relative to an $\mathcal{M}$-computation on some palindrome with prefix $y-$, the crossing sequence on cell $|y|$ is $C S_{i}$.

This can be seen as follows. Suppose $y \neq x_{1} x_{2} \ldots x_{i}$ leads to the same crossing sequence $C S_{i}$ on cell $|y|$ for an $\mathcal{M}$-computation on some palindrome $y z z^{R} y^{R}$. Run $\mathcal{M}$ on input $y x_{i+1} \ldots x_{n} x^{R}$. Consider the behaviour of $\mathcal{M}$ while the head is on the left part $y$. This behaviour is exactly the same as that for the run on input $y z z^{R} y^{R}$ because the sole useful information for $\mathcal{M}$ while scanning $y$ comes from the crossing sequence at cell $|y|$. In particular, $\mathcal{M}$ - which halts on cell 1 - accepts this input $y x_{i+1} \ldots x_{n} x^{R}$. But this is not a palindrome! Contradiction. 
Observe that the way $x_{1} x_{2} \ldots x_{i}$ is uniquely determined by $C S_{i}$ is quite complex. But we don't care about that. It will just charge the $O(1)$ constant in (11).

Using Proposition 0 with the binary string associated to $C S_{i}$ which is $c$ times longer (where $c=\lceil|Q|\rceil,|Q|$ being the number of states), we see that

$$
K\left(x_{1} x_{2} \ldots x_{i}\right) \leq c\left|T_{i}\right|+O(1)
$$

If $i \geq \frac{n}{2}$ then $x_{1} x_{2} \ldots x_{\frac{n}{2}}$ is uniquely determined by the pair $\left(x_{1} x_{2} \ldots x_{i}, \frac{n}{2}\right)$. Hence also by the pair $\left(C S_{i}, \frac{n}{2}\right)$. Since the binary representation of $\frac{n}{2}$ uses $\leq \log (n)$ bits, this pair can be encoded with $2 c\left|C S_{i}\right|+\log (n)+1$ bits. Thus,

$$
K\left(x_{1} x_{2} \ldots x_{\frac{n}{2}}\right) \leq 2 c\left|C S_{i}\right|+\log (n)+O(1)
$$

Now, let's sum equations (12) for $i=\frac{n}{2}, \ldots, n$. Observe that the sum of the lengthes of the crossing sequences $C S_{\frac{n}{2}}, \ldots, C S_{n}$ is at most the number $T$ of computation steps. Therefore, this summation leads to

$$
\frac{n}{2} K\left(x_{1} x_{2} \ldots x_{\frac{n}{2}}\right) \leq 2 c T+\frac{n}{2} \log (n)+O\left(\frac{n}{2}\right)
$$

Now, consider as $x$ a string such that $x_{1} x_{2} \ldots x_{\frac{n}{2}}$ is incompressible, i.e. $K\left(x_{1} x_{2} \ldots x_{\frac{n}{2}}\right) \geq \frac{n}{2}$. Equation (13) leads to

$$
\left(\frac{n}{2}\right)^{2} \leq 2 c T+\frac{n}{2} \log (n)+O\left(\frac{n}{2}\right)
$$

whence $T \geq O\left(n^{2}\right)$. Since the input $x x^{R}$ has length $2 n$, this proves the quadratic lower bound. QED

\section{Prefix complexity}

\subsection{Self delimiting programs}

Q: I heard about prefix complexity. What is it?

A: Prefix complexity is a very interesting variant of Kolmogorov complexity which was introduced around 1973 by Levin [28] and, independently, by Chaitin [8].

The basic idea is taken from some programming languageswhich have an explicit delimiter to mark the end of a program. For instance, $P A S C A L$ uses "end.". Thus, no program can be a proper prefix of another program.

Q: This is not true with PROLOG programs: you can always add a new clause. 
A: To execute a PROLOG program, you have to write down a query. And the end of a query is marked by a full stop. So, it's also true for PROLOG.

Q: OK. However, it's not true for $C$ programs nor $L I S P$ programs.

A: Hum... You are right.

\subsection{Chaitin-Levin prefix complexity}

A: Let's say that a set $X$ of strings is prefix-free if no string in $X$ is a proper prefix of another string in $X$. A programming language $A:\{0,1\}^{*} \rightarrow\{0,1\}^{*}$ is prefix if its domain is a prefix-free set.

Q: So the programming language $P A S C A L$ that you seem to be fond of is prefix.

A: Sure, $P A S C A L$ is prefix.

A: But, what's new with this special condition?

A: Kolmogorov Invariance Theorem from $\$ 2.1$ goes through with prefix programming languages, leading to the prefix variant $H$ of $K$.

Theorem 17 (Invariance theorem). There exists a prefix partial computable function $U^{\text {prefix }}:\{0,1\}^{*} \rightarrow\{0,1\}^{*}$. such that $K_{U^{\text {prefix }}} \leq K_{A}+O(1)$ for any prefix partial computable function $A:\{0,1\}^{*} \rightarrow\{0,1\}^{*}$. In other words, up to an additive constant, $K_{U^{p r e f i x}}$ is the smallest one among the $K_{A}$ 's.

Definition 18 (Prefix Kolmogorov complexity). Prefix Kolmogorov complexity $H:\{0,1\}^{*} \rightarrow \mathbb{N}$ is any fixed such function $K_{U^{p r e f i x}}$.

\subsection{Comparing $K$ and $H$}

Q: How does $H$ compare to $K$ ?

A: A simple relation is as follows:

Proposition 19. $K(x)-O(1) \leq H(x) \leq K(x)+2 \log (K(x))+O(1)$ Idem with $K(\mid)$ and $H(\mid)$.

The first inequality is a mere application of the Invariance Theorem for $K$ (since $U^{\text {prefix }}$ is a programming language). To get the second one, we consider a programming language $U$ such that $K=K_{U}$ and construct a prefix programming language $U^{\prime}$ as follows: the domain of $U^{\prime}$ is the set of strings of the form $\operatorname{pad}(|p|) 1 p$ and $U^{\prime}(\operatorname{pad}(|p|) 1 p)=U(p)$. By very construction, 
the domain of $U^{\prime}$ is prefix-free. Also, $K_{U^{\prime}}(x)=K_{U}(x)+2 \log \left(K_{U}(x)\right)+1$. An application of the Invariance Theorem for $H$ gives the second inequality of the Proposition.

This inequality can be improved. A better encoding leads to

$$
H(x) \leq K(x)+\log (K(x))+2 \log \log (K(x))+O(1)
$$

Sharper relations have been proved by Solovay, 1975 (unpublished [43], cf. also [30] p. 211):

Proposition 20. $H(x)=K(x)+K(K(x))+O(K(K(K(x))))$

$$
K(x)=H(x)-H(H(x))-O(H(H(H(x))))
$$

\subsection{How big is $H$ ?}

Q: How big is $H$ ?

A: $K$ and $H$ behave in similar ways. Nevertheless, there are some differences. Essentially a logarithmic term.

Proposition 21. $H(x) \leq|x|+2 \log (|x|)+O(1)$

To prove it, apply the $H$ Invariance Theorem to the prefix function

$$
A(\operatorname{pad}(|x|) 1 x)=x \text {. }
$$

Of course, it can be improved to

$$
H(x) \leq|x|+\log (|x|)+2 \log \log (|x|)+O(1)
$$

Q: How big can be $H(x)-|x|$ ?

A: Well, to get a non trivial question, we have to fix the length of the $x$ 's. The answer is not a simple function of $|x|$ as expected, it does use $H$ itself:

$$
\max _{|x|=n}(H(x)-|x|)=H(|x|)+O(1)
$$

Q: How big can be $H(x)-K(x)$ ?

A: It can be quite large:

$$
K(x) \leq|x|-\log (|x|) \leq|x| \leq H(x)
$$

happens for arbitrarily large $x$ 's ([30] Lemma 3.5.1 p. 208).

\subsection{Convergence of series and the Coding Theorem}

Q: What's so really special with this prefix condition?

A: The possibility to use Kraft's inequality. This inequality tells you that if $Z$ is a prefix-free set of strings then $\Sigma_{p \in Z} 2^{-|p|} \leq 1$. 
Kraft's inequality is not hard to prove. Denote $I_{u}$ the set of infinite strings which admit $u$ as prefix. Observe that

1) $2^{-|p|}$ is the probability of $I_{u}$.

2) If $u, v$ are prefix incomparable then $I_{u}$ and $I_{v}$ are disjoint.

3) Since $Z$ is prefix, the $I_{u}$ 's, $u \in Z$ are pairwise disjoint and their union has probability $\Sigma_{p \in Z} 2^{-|p|}<1$

The $K_{A}(x)$ 's are lengthes of distinct programs in a prefix set (namely, the domain of $A$ ). So, Kraft's inequality implies

$$
\Sigma_{x \in\{0,1\} * 2^{-K_{A}(x)}<1 .}
$$

In fact, $H$ satisfies the following very important property, proved by Levin 229] (which can be seen as another version of the Invariance Theorem for $H)$ :

Theorem 22 (Coding Theorem). Up to a multiplicative factor, $2^{-H}$ is maximum among functions $F:\{0,1\}^{*} \rightarrow \mathbf{R}$ such that $\Sigma_{x \in\{0,1\}^{*}} F(x)<+\infty$ and which are approximable from below (in a sense dual to that in \$3.1, i.e. the set of pairs $(x, q)$ such that $q$ is rational and $q<F(x)$ is c.e.).

\section{Random infinite sequences}

\subsection{Top-down approach to randomness of infinite sequences}

Q: So, we now come to random infinite sequences.

A: It happens that there are two equivalent ways to get a mathematical notion of random sequences. We shall first consider the most natural one, which is a sort of "top-down approach".

Probability laws tell you that with probability one such and such things happen, i.e. that some particular set of sequences has probability one. A natural approach leads to consider as random those sequences which satisfy all such laws, i.e. belong to the associated sets (which have probability one).

An easy way to realize this would be to declare a sequence to be random just in case it belongs to all sets (of sequences) having probability one or, equivalently, to no set having probability zero. Said otherwise, the family of random sequences would be the intersection of all sets having probability one, i.e. the complement of the union of all sets having probability zero.

Unfortunately, this family is empty! In fact, let $r$ be any sequence: the singleton set $\{r\}$ has probability zero and contains $r$.

In order to maintain the idea, we have to consider a not too big family of sets with probability one. 
Q: A countable family.

A: Right. The intersection of a countable family of set with probability one will have probability one. So that the set of random sequences will have probability one, which is a much expected property.

\subsection{Frequency tests and von Mises random sequences}

A: This top-down approach was pionneered by Richard von Mises in 1919 (48, 49 ) who insisted on frequency statistical tests. He declared an infinite binary sequence $a_{0} a_{1} a_{2} \ldots$ to be random (he used the term Kollektiv) if the frequence of 1 's is "everywhere" fairly distributed in the following sense:

i) Let $S_{n}$ be the number of 1's among the first $n$ terms of the sequence. Then $\lim _{n \rightarrow \infty} \frac{S_{n}}{n}=\frac{1}{2}$.

ii) The same is true for every subsequence $a_{n_{0}+1} a_{n_{1}+1} a_{n_{2}+1} \ldots$ where $n_{0}, n_{1}, n_{2} \ldots$ are the successive integers $n$ such that $\phi\left(a_{0} a_{1} \ldots a_{n}\right)=1$ where $\phi$ is an "admissible" place-selection rule.

What is an "admissible" place-selection rule was not definitely settled by von Mises. Alonzo Church, 1940, proposed that admissibility be exactly computability.

It is not difficult to prove that the family of infinite binary sequence satisfying the above condition has probability one for any place-selection rule. Taking the intersection over all computable place-selection rules, we see that the family of von Mises-Church random sequences has probability one.

However, von Mises-Church notion of random sequence is too large. There are probability laws which do not reduce to tests with place-selection rules and are not satisfied by all von Mises-Church random sequences. As shown by Jean Ville, 47] 1939, this is the case for the law of iterated logarithm. This very important law (due to A. I. Khintchin, 1924) expresses that with probabililty one

$$
\lim \sup _{n \rightarrow+\infty} \frac{S_{n}^{*}}{\sqrt{2 \log \log (n)}}=1 \text { and } \lim \inf _{n \rightarrow+\infty} \frac{S_{n}^{*}}{\sqrt{2 \log \log (n)}}=-1
$$

where $S_{n}^{*}=\frac{S_{n}-\frac{n}{2}}{\sqrt{\frac{n}{4}}}$ (cf. William Feller's book [14], p. 186, 204-205).

Q: Wow! What do these equations mean?

A: They are quite meaningful. The quantities $\frac{n}{2}$ and $\sqrt{\frac{n}{4}}$ are the expectation and standard deviation of $S_{n}$. So that, $S_{n}^{*}$ is obtained from $S_{n}$ by 
normalization: $S_{n}$ and $S_{n}^{*}$ are linearly related as random variables, and $S_{n}^{*}$ 's expectation and standard deviation are 0 and 1.

Let's interpret the limsup equation, the other one being similar (in fact, it can be obtained from the first one by symmetry).

Remember that $\lim \sup _{n \rightarrow+\infty} f_{n}$ is obtained as follows. Consider the sequence $v_{n}=\sup _{m \geq n} f_{m}$. The bigger is $n$ the smaller is the set $\{m: m \geq n\}$. So that the sequence $v_{n}$ decreases, and $\lim \sup _{n \rightarrow+\infty} f_{n}$ is its limit.

The law of iterated logarithm tells you that with probabililty one the set $\left\{n: S_{n}^{*}>\lambda \sqrt{2 \log \log (n)}\right\}$ is finite in case $\lambda>1$ and infinite in case $\lambda<1$.

Q: OK.

A: More precisely, there are von Mises-Church random sequences which satisfy $\frac{S_{n}}{n} \geq \frac{1}{2}$ for all $n$, a property which is easily seen to contradict the law of iterated logarithm.

Q: So, von Mises' approach is definitely over.

A: No. Kolmogorov, 1963 [22], and Loveland, 1966 [31], independently considered an extension of the notion of place-selection rule.

Q: Kolmogorov once more...

A: Indeed. Kolmogorov allows place-selection rules giving subsequences proceeding in some new order, i.e. mixed subsequences. The associated notion of randomness is called Kolmogorov stochastic randomness (cf. [26] 1987). Since there are more conditions to satisfy, stochastic random sequences form a subclass of von Mises-Church random sequences. They constitute, in fact, a proper subclass ([31]).

However, it is not known whether they satisfy all classical probability laws.

\subsection{Martin-Löf random sequences}

Q: So, how to come to a successful theory of random sequences?

A: Martin-Löf found such a theory.

Q: That was not Kolmogorov? The same Martin-Löf you mentioned concerning random finite strings?

A: Yes, the same Martin-Löf, in the very same paper [34] in 1965. Kolmogorov looked for such a notion, but it was Martin-Löf, a Swedish mathematician, who came to the pertinent idea. At that time, he was a pupil of Kolmogorov and studied in Moscow. Martin-Löf made no use of Kolmogorov random finite string to get the right notion of infinite random sequence. 
What he did is to forget about the frequency character of computable statistical tests (in von Mises-Church notion of randomness) and look for what could be the essence of general statistical tests and probability laws. Which he did both for finite strings and for infinite sequences.

Q: Though intuitive, this concept is rather vague!

A: Indeed. And Martin-Löf's analysis of what can be a probability law is quite interesting.

To prove a probability law amounts to prove that a certain set $X$ of sequences has probability one. To do this, one has to prove that the exception set - which is the complement $Y=\{0,1\}^{\mathbb{N}} \backslash X$ - has probability zero. Now, in order to prove that $Y \subseteq\{0,1\}^{\mathbb{N}}$ has probability zero, basic measure theory tells us that one has to include $Y$ in open sets with arbitrarily small probability. I.e. for each $n \in \mathbb{N}$ one must find an open set $U_{n} \supseteq Y$ which has probability $\leq \frac{1}{2^{n}}$.

If things were on the real line $\mathbf{R}$ we would say that $U_{n}$ is a countable union of intervals with rational endpoints.

Here, in $\{0,1\}^{\mathbb{N}}, U_{n}$ is a countable union of sets of the form $I_{u}=u\{0,1\}^{\mathbb{N}}$ where $u$ is a finite binary string and $I_{u}$ is the set of infinite sequences which extend $u$. Well, in order to prove that $Y$ has probability zero, for each $n \in \mathbb{N}$ one must find a family $\left(u_{n, m}\right)_{m \in \mathbb{N}}$ such that $Y \subseteq \bigcup_{m} I_{u_{n, m}}$ and $\operatorname{Proba}\left(\bigcup_{m} I_{u_{n, m}}\right) \leq \frac{1}{2^{n}}$ for each $n \in \mathbb{N}$.

And now Martin-Löf makes a crucial observation: mathematical probability laws which we can consider necessarily have some effective character. And this effectiveness should reflect in the proof as follows:

the doubly indexed sequence $\left(u_{n, m}\right)_{n, m \in \mathbb{N}}$ is computable.

Thus, the set $\bigcup_{m} I_{u_{n, m}}$ is a computably enumerable open set and $\bigcap_{n} \bigcup_{m} I_{u_{n, m}}$ is a countable intersection of a computably enumerable family of open sets.

Q: This observation has been checked for proofs of usual probability laws?

A: Sure. Let it be the law of large numbers, that of iterated logarithm... In fact, it's quite convincing.

Q: This open set $\bigcup_{m} I_{u_{n, m}}$ could not be computable?

A: No. A computable set in $\{0,1\}^{\mathbb{N}}$ is always a finite union of $I_{u}$ 's.

Q: Why?

A: What does it mean that $Z \subseteq\{0,1\}^{\mathbb{N}}$ is computable? That there is some Turing machine such that, if you write an infinite sequence $\alpha$ on the input 
tape then after finitely many steps, the machine tells you if $\alpha$ is in $Z$ or not. When it does answer, the machine has read but a finite prefix $u$ of $\alpha$, so that it gives the same answer if $\alpha$ is replaced by any $\beta \in I_{u}$. In fact, an application of König's lemma (which we shall not detail) shows that we can bound the length of such a prefix $u$. Whence the fact that $Z$ is a finite union of $I_{u}$ 's.

Q: OK. So, we shall take as random sequences those sequences which are outside any set which is a countable intersection of a computably enumerable family of open sets and has probability zero.

A: This would be too much. Remember, $\operatorname{Proba}\left(\bigcup_{m} I_{u_{n, m}}\right) \leq \frac{1}{2^{n}}$. Thus, the way the probability of $\bigcup_{m} I_{u_{n, m}}$ tends to 0 is computably controlled.

So, here is Martin-Löf's definition:

Definition 23. A set of infinite binary sequences is constructively of probability zero if it is included in $\bigcap_{n} \bigcup_{m} I_{u_{n, m}}$ where $(m, n) \mapsto u_{n, m}$ is a partial computable function $\mathbb{N}^{2} \rightarrow\{0,1\}^{*}$ such that $\operatorname{Proba}\left(\bigcup_{m} I_{u_{n, m}}\right) \leq \frac{1}{2^{n}}$ for all $n$.

And now comes a very surprising theorem (Martin-Löf, [34], 1966):

Theorem 24. There is a largest set of sequences (for the inclusion ordering) which is constructively of probability zero.

Q: Largest? up to what?

A: Up to nothing. Really largest set: it is constructively of probability zero and contains any other set constructively of probability zero.

Q: How is it possible?

A: Via a diagonalization argument. The construction has some technicalities but we can sketch the ideas. From the well-known existence of universal c.e. sets, we get a computable enumeration $\left(\left(O_{i, j}\right)_{i}\right)_{j}$ of sequences of c.e. open sets. A slight transformation allows to satisfy the inequality $\operatorname{Proba}\left(O_{i}\right) \leq \frac{1}{2^{2}}$. Now, set $U_{j}=\bigcup_{e} O_{e, e+j+1}$ (here lies the diagonalization!) Clearly, $\operatorname{Proba}\left(O_{j}\right) \leq \sum_{e} \frac{1}{2^{e+j+1}}=\frac{1}{2^{j}}$, so that $\bigcap_{j} U_{j}$ is constructively of probability zero. Also, $U_{j} \supseteq O_{i, j}$ for all $j \geq i$ whence $\left(\bigcap_{j} U_{j}\right) \supseteq\left(\bigcap_{j} O_{i, j}\right)$.

Q: So, Martin-Löf random sequences are exactly those lying in this largest set.

A: Yes. And all theorems in probability theory can be strengthened by replacing "with probability one" by "for all Martin-Löf random sequences" 


\subsection{Bottom-up approach to randomness of infinite sequences: Martin-Löf's Large oscillations theorem}

Q: So, now, what is the bottom-up approach?

A: This approach looks at the asymptotic algorithmic complexity of the prefixes of the infinite binary sequence $a_{0} a_{1} a_{2} \ldots$, namely the $K\left(a_{0} \ldots a_{n}\right)$ 's.

The next theorem is the first significative result relevant to this approach. Point 2 is due to Albert Meyer and Donald Loveland, 1969 [32] p. 525. Points 3,4 are due to Gregory Chaitin, 1976 [9]. (Cf. also [30] 2.3.4 p.124).

Theorem 25. The following conditions are equivalent:

1) $a_{0} a_{1} a_{2} \ldots$ is computable

2) $K\left(a_{0} \ldots a_{n} \mid n\right)=O(1)$.

3) $\left|K\left(a_{0} \ldots a_{n}\right)-K(n)\right| \leq O(1)$.

4) $\left|K\left(a_{0} \ldots a_{n}\right)-\log (n)\right| \leq O(1)$.

Q: Nice results. Let me tell what I see. We know that $K(x) \leq|x|+O(1)$. Well, if we have the equality, $K\left(a_{0} \ldots a_{n}\right)=n-O(1)$, i.e. if maximum complexity occurs for all prefixes, then the sequence $a_{0} a_{1} a_{2} \ldots$ should be random! Is it indeed the case?

A: That's a very tempting idea. And Kolmogorov had also looked for such a characterization. Unfortunately, as Martin-Löf proved around 1965 (1966, [35]), there is no such sequence! It is a particular case of a more general result (just set $\mathrm{f}(\mathrm{n})=$ constant).

Theorem 26 (Large oscillations, [35]). Let $f: \mathbb{N} \rightarrow \mathbb{N}$ be a computable function such that $\Sigma_{n \in \mathbb{N}} 2^{-f(n)}=+\infty$. Then, for every binary sequence $a_{0} a_{1} a_{2} \ldots$ there are infinitely many n's such that $K\left(a_{0} \ldots a_{n} \mid n\right)<n-f(n)$.

Q: So, the bottom-up approach completely fails as concerns a characterization of random sequences. Hum... But it does succeed as concerns computable sequences, which were already fairly well characterized. Funny!

A: It's however possible to sandwich the set of Martin-Löf random sequences between two sets of probability one defined in terms of the $K$ complexity of prefixes.

Theorem 27 ([35]). Let $f: \mathbb{N} \rightarrow \mathbb{N}$ be computable such that the series $\Sigma 2^{-f(n)}$ is computably convergent. Set

$$
\begin{aligned}
X & =\left\{a_{0} a_{1} \ldots: K\left(a_{0} \ldots a_{n} \mid n\right) \geq n-O(1) \text { for infinitely many } n ' s .\right\} \\
Y_{f} & =\left\{a_{0} a_{1} \ldots: K\left(a_{0} \ldots a_{n} \mid n\right) \geq n-f(n) \text { for all but finitely many } n ' s .\right\}
\end{aligned}
$$


Denote $M L$ the set of Martin-Löf random sequences. Then $X$ and $Y_{f}$ have probability one and $X \subset M L \subset Y_{f}$.

NB: Proper inclusions have been proved by Peter Schnorr, 1971 [39] (see also [30] 2.5.15 p.154).

Let's illustrate this theorem on an easy and spectacular corollary which uses the fact that $2^{-2 \log (n)}=\frac{1}{n^{2}}$ and that the series $\Sigma \frac{1}{n^{2}}$ is computably convergent: if $K\left(a_{0} \ldots a_{n} \mid n\right) \geq n-c$ for infinitely many $n$ 's then $K\left(a_{0} \ldots a_{n} \mid\right.$ $n) \geq n-2 \log (n)$ for all but finitely many $n$ 's.

\subsection{Bottom-up approach with prefix complexity}

Q: What about considering prefix Kolmogorov complexity?

A: Kolmogorov's original idea does work with prefix Kolmogorov complexity. This has been proved by Claus Peter Schnorr (1974, unpublished, cf. [8] Remark p. 106, and 10] p. 135-137 for a proof).

Robert M. Solovay, 1974 (unpublished [43]) strengthened Schnorr's result (cf. 10] p. 137-139).

Theorem 28. The following conditions are equivalent:

1) $a_{0} a_{1} a_{2} \ldots$ is Martin-Löf ramdom random

2) $H\left(a_{0} \ldots a_{n}\right) \geq n-O(1)$ for all $n$.

3) $\lim _{n \rightarrow+\infty}\left(H\left(a_{0} \ldots a_{n}\right)-n\right)=+\infty$.

4) For any c.e. sequence $\left(A_{i}\right)_{i}$ of open subsets of $\{0,1\}^{\mathbb{N}}$ if $\Sigma_{i} \operatorname{Proba}\left(A_{i}\right)<$ $+\infty$ then $a_{0} a_{1} a_{2} \ldots$ belongs to finitely many $A_{i}$ 's.

These equivalences stress the robustness of the notion of Martin-Löf ramdom sequence.

\subsection{Top-down/Bottom-up approaches: a sum up}

Q: I get somewhat confused with these two approaches. Could you sum up.

A: The top-down and bottom-up approaches both work and lead to the very same class of random sequences.

Kolmogorov looked at the bottom-up approach from the very beginning in 1964. But nothing was possible with the original Kolmogorov complexity, Levin-Chaitin's variant $H$ was needed.

Q: Ten years later...

A: As for the top-down approach, it was pionneered by von Mises since 1919 and made successful by Martin-Löf in 1965. Martin-Löf had to give 
up von Mises frequency tests. However, Kolmogorov was much interested by these frequency tests ([22]), and he refined them in a very clever way with the purpose to recover Martin-Löf randomness, which lead him to the notion of Kolmogorov stochastic randomness. Unfortunately, up to now, we only know that

Martin-Löf random $\Rightarrow$ stochastic random $\Rightarrow$ von Mises-Church random. The second implication is known to be strict but not the first one. Would it be an equivalence, this would give a quite vivid characterization of random sequences via much concrete tests.

\subsection{Randomness with other probability distributions}

Q: All this is relative to the uniform probability distribution. Can it be extended to arbitrary probability distributions?

A: Not arbitrary probability distributions, but computable Borel ones: those distributions $P$ such that the sequence of reals $\left(P\left(I_{u}\right)\right)_{u \in\{0,1\}^{*}}$ (where $I_{u}$ is the set of infinite sequences which extend $\left.u\right)$ is computable, i.e. there is a computable function $f:\{0,1\}^{*} \times \mathbb{N} \rightarrow \mathbf{Q}$ such that

$$
\left|P\left(I_{u}\right)-f(u, n)\right| \leq \frac{1}{2^{n}} .
$$

Martin-Löf's definition of random sequences extends trivially. As for characterizations with variants of Kolmogorov complexity, one has to replace the length of a finite string $u$ by the quantity $-\log \left(P\left(I_{u}\right)\right)$.

\subsection{Chaitin's real $\Omega$}

Q: I read a lot of things about Chaitin's real $\Omega$.

A: Gregory Chaitin, 1987 [11], explicited a spectacular random real and made it very popular.

Consider a universal prefix partial recursive function $U$ and let $\Omega$ be the Lebesgue measure of the set

$$
\left\{\alpha \in\{0,1\}^{\mathbb{N}} \mid \exists n U(\alpha\lceil n) \text { is defined }\}\right.
$$

Q: Seems to be an avatar of the halting problem.

A: Indeed. It is the probability that, on an infinite input, the machine which computes $U$ halts in finite time (hence after reading a finite prefix of its input).

$$
\Omega=\Sigma\left\{2^{-|p|} \mid U \text { halts on input } p\right\}
$$

Theorem 29. The binary expansion of $\Omega$ is Martin-Löf ramdom. 
Q: How does one prove that $\Omega$ is ramdom?

A: $U$ has prefix-free domain, hence $\Omega=\Sigma\left\{2^{-|p|} \mid p \in \operatorname{domain}(U)\right\}<1$. Any halting program with length $n$ contributes for exactly $2^{-n}$ to $\Omega$. Thus, if you know the first $k$ digits of $\Omega$ then you know the number of halting programs with length $\leq k$. From this number, by dovetailing, you can get the list of the halting programs with length $\leq k$ (cf. $\$ 3.1$, 3.2). Having these programs, you can get the first string $u$ which is not the output of such a program. Clearly, $H(u)>k$. Now, $u$ is computably obtained from the first $k$ digits of $\Omega$, so that by Proposition 0 we have $H(u) \leq H\left(\omega_{0} \ldots \omega_{k}\right)+$ $O(1)$. Whence $H\left(\omega_{0} \ldots \omega_{k}\right) \geq k+O(1)$, which is condition 2 of Theorem 28 (Schnorr condition). This proves that the binary expansion of $\Omega$ is a Martin-Löf ramdom sequence.

Q: $\Omega$ seems to depend on the universal machine.

A: Sure. We can speak of the class of Chaitin $\Omega$ reals: those reals which express the halting probability of some universal prefix programming language.

Cristian Calude \& Peter Hertling \& Bakhadyr Khoussainov \& Yongge Wang, 1998 [4] (cf. also Antonìn Kučera \& Theodore Slaman, 2000 [27]) proved a very beautiful result: $r$ is a Chaitin $\Omega$ real if and only if (the binary development of $r$ is Martin-Löf random and $r$ computably enumerable from below (i.e. the set of rational numbers $<r$ is c.e.).

Q: I read that this real has incredible properties.

A: This real has a very simple and appealing definition. Moreover, as we just noticed, there is a simple way to get all size $n$ halting programs from its $n$ first digits. This leads to many consequences due to the following fact: any $\Sigma_{1}^{0}$ statement of the form $\exists \vec{x} \Phi(\vec{x})$ (where $\Phi$ is a computable relation) is equivalent to a statement insuring that a certain program halts, and this program is about the same size as the statement. Now, deciding the truth of $\Sigma_{1}^{0}$ statements is the same as deciding that of $\Pi_{1}^{0}$ statements.

And significant $\Pi_{1}^{0}$ statements abound! Like Fermat's last theorem (which is now Wiles' theorem) or consistency statements. This is why Chaitin says $\Omega$ is the "Wisdom real".

Other properties of $\Omega$ are common to all reals which have Martin-Löf ramdom binary expansions. For instance, transcendance and the fact that any theory can give us but finitely many digits.

Hum... About that last point, using Kleene's recursion theorem, Robert Solovay, 1999 [45], proved that there are particular Chaitin $\Omega$ reals about which a given theory can not predict any single bit! 


\subsection{Non computable invariance}

Q: In some sense, Martin-Löf ramdomness is a part of recursion theory. Do random sequences form a Turing degree or a family of Turing degrees?

A: Oh, no! Randomness is definitely not computable invariant. It's in fact a very fragile notion: quite insignificant modifications destroy randomness. This makes objects like $\Omega$ so special.

Let's illustrate this point on an example. Suppose you transform a random sequence $a_{0} a_{1} a_{2} a_{3} \ldots$ into $a_{0} 0 a_{1} 0 a_{2} 0 a_{3} 0 \ldots$ The sequence you obtain has the same Turing degree as the original one, but it is no more random since its digits with odd ranks are all 0. A random sequence has to be random everywhere. Hum ... for Martin-Löf random reals, I should rather say "every c.e. where".

Q: Everywhat?

A: "Every c.e. where". I mean that if $f$ is a computable function from $\mathbb{N}$ into $\mathbb{N}$ (in other words, a computable enumeration of an c.e. set) then the sequence of digits with ranks $f(0), f(1), f(2), \ldots$ of a Martin-Löf random sequence has to be Martin-Löf random. In fact, you recognize here an extraction process à la von Mises for which a random sequence should give another random sequence.

Q: OK. What about many-one degrees?

A: Same. Let's represent a binary infinite sequence $\alpha$ by the set $X_{\alpha}$ of positions of digits 1 in $\alpha$. Then,

$$
n \in X_{a_{0} a_{1} a_{2} \ldots} \Leftrightarrow 2 n \in X_{a_{0} 0 a_{1} 0 a_{2} 0 \ldots}
$$

Also, let $\varphi(2 n)=n$ and $\varphi(2 n+1)=k$ where $k$ is some fixed rank such that $a_{k}=0$, then

$$
n \in X_{a_{0} 0 a_{1} 0 a_{2} 0 l d o t s} \Leftrightarrow \varphi(n) \in X_{a_{0} a_{1} a_{2} \ldots}
$$

These two equivalences prove that $X_{a_{0} a_{1} a_{2} \ldots}$ and $X_{a_{0} 0 a_{1} 0 a_{2} 0 \ldots}$ are many-one equivalent.

\section{More randomness}

There are more things in heaven and earth, Horatio, Than are dreamt of in your philosophy.

Hamlet, William Shakespeare 


\subsection{Beyond c.e.: oracles and infinite computations}

Q: Are there other random reals than Chaitin $\Omega$ reals ?

A: Sure. Just replace in Martin-Löf's definition the computable enumerability condition by a more complex one. For instance, you can consider $\Sigma_{2}^{0}$ sets, which amounts to computable enumerability with oracle $\emptyset^{\prime}$ (the set which encodes the halting problem for Turing machines).

Q: Wait, wait. Just a minute ago, you said that for all classical probability laws, c.e. open sets, i.e. $\Sigma_{1}^{0}$ sets, are the ones which come in when proving that the exception set to the law has probability zero. So, what could be the use of such generalizations?

A: Clearly, the more random sequences you have which satisfy classical probability laws, the more you strengthen these theorems as we said earlier. In this sense, it is better to stick to Martin-Löf's definition. But you can also want to consider random sequences as worst objects to use in some context. Depending on that context, you can be lead to ask for much complex randomness conditions.

Also, you can have some very natural objects much alike Chaitin real $\Omega$ which can be more complex.

Q: Be kind, give an example!

A: In a recent paper, 2001 [2], Verónica Becher \& Chaitin \& Sergio Daicz consider the probability that a prefix universal programming language produces a finite output, though possibly running indefinitely. They prove that this probability is an $\Omega^{\prime}$ Chaitin real, i.e. its binary expansion is $\emptyset^{\prime}$-random. Becher \& Chaitin, 2002 [1], consider the probability for the output to represent a cofinite set of integers, relatively to some coding of sets of integers by sequences. They prove it to be an $\Omega^{\prime \prime}$ Chaitin real.

Such reals are as much appealing and remarkable as Chaitin's real $\Omega$ and also they are logically more complex.

\subsection{Far beyond: Solovay random reals in set theory}

Q: I heard about Solovay random reals in set theory. Has it anything to do with Martin-Löf random reals?

A: Hum... These notions come from very different contexts. But well, there is a relation: proper inclusion. Every Solovay random real is MartinLöf random. The converse being far from true. In fact, these notions of randomness are two extreme notions of randomness. Martin-Löf randomness 
is the weakest condition whereas Solovay randomness is really the strongest one. So big indeed that for Solovay reals you need to work in set theory, not merely in recursion theory, and even worse, you have to consider two models of set theory, say $M_{1}$ and an inner submodel $M_{2}$ with the same ordinals...

Q: You mean transfinite ordinals?

A: Yes, $0,1,2,3, \ldots, \omega, \omega+1, \omega+2, \ldots, \omega+\omega$ (which is $\omega .2$ ) an so on: $\omega .3, \ldots, \omega \cdot \omega\left(\right.$ which is $\left.\omega^{2}\right), \ldots, \omega^{3}, \ldots, \omega^{\omega}, \ldots$

In a model of set theory, you have reals and may consider Borel sets, i.e. sets obtained from rational intervals via iterated countable unions and countable intersections.

Thus, you have reals in $M_{1}$ and reals in $M_{2}$ and every $M_{2}$ real is also in $M_{1}$. You also have Borel sets defined in $M_{2}$. And to each such Borel set $X_{2}$ corresponds a Borel set $X_{1}$ in $M_{1}$ with the same definition (well, some work is necessary to get a precise meaning, but it's somewhat intuitive). One can show that $X_{2} \subseteq X_{1}$ and that $X_{1}, X_{2}$ have the very same measure, which is necessarily a real in $M_{2}$. Such a Borel set $X_{1}$ in $M_{1}$ will be called a $M_{2}$-coded Borel set.

Now, a real $r$ in $M_{1}$ is Solovay random over $M_{2}$ if it lies in no measure zero $M_{2}$-coded Borel set of $M_{1}$. Such a real $r$ can not lie in the inner model $M_{2}$ because $\{r\}$ is a measure zero Borel set and if $r$ were in $M_{2}$ then $\{r\}$ would be $M_{2}$-coded and $r$ should be outside it, a contradiction.

In case $M_{1}$ is big enough relative to $M_{2}$ it can contain reals which are Solovay random over $M_{2}$. It's a rather tough subject, but you see:

- Martin-Löf random reals are reals outside all c.e. $G_{\delta}$ sets (i.e. intersection of an c.e. sequence of open sets) constructively of measure zero. In other words, outside a very smooth countable family of Borel sets. Such Borel sets are, in fact, coded in any inner submodel of set theory.

- Solovay random reals over a submodel of set theory are reals outside every measure zero Borel set coded in that submodel. Thus Solovay random reals can not be in the inner submodel. They may or may not exist, depending on how big is $M_{1}$ relative to $M_{2}$.

Q: What a strange theory. What about the motivations?

A: Solovay introduced random reals in set theory at the pionneering time of independence results in set theory, using the method of forcing invented by Paul J. Cohen. That was in the 60's. He used them to get a model of set theory in which every set of reals is Lebesgue measurable [44].

Q: Wow! it's getting late. 
A: Hope you are not exhausted.

Q: I really enjoyed talking with you on such a topic.

Note. The best reference to the subject are

- Li \& Vitanyi's book 30

- Downey \& Hirschfeldt's book 13]

Caution: In these two books, $C, K$ denote what is here - and in many papers - denoted $K, H$.

Among other very useful references: [3], [12], [17], [40] and [46].

Gregory Chaitin's papers are available on his home page.

\section{References}

[1] V. Becher and G. Chaitin. Another example of higher order randomness. Fund. Inform., 51(4):325-338, 2002.

[2] V. Becher, G. Chaitin, and S. Daicz. A highly random number. In C.S. Calude, M.J. Dineen, and S. Sburlan, editors, Proceedings of the Third Discrete Mathematics and Theoretical Computer Science Conference (DMTCS'01), pages 55-68. Springer-Verlag, 2001.

[3] C. Calude. Information and randomness. Springer, 1994.

[4] C.S. Calude, P.H. Hertling, and B. Khoussainov Y. Wang. Recursively enumerable reals and Chaitin $\Omega$ numbers. In STACS 98 (Paris, 1998), number 1373 in Lecture Notes in Computer Science, pages 596-606. Springer-Verlag, 1998.

[5] G. Chaitin. On the length of programs for computing finite binary sequences. J. Assoc. Comput. Mach., 13:547-569, 1966.

[6] G. Chaitin. On the length of programs for computing finite binary sequences: statistical considerations. J. Assoc. Comput. Mach., 16:145159, 1969.

[7] G. Chaitin. Computational complexity and Gdel incompleteness theorem. ACM SIGACT News, 9:11-12, 1971. Available on Chaitin's home page. 
[8] G. Chaitin. A theory of program size formally identical to information theory. Journal of the ACM, 22:329-340, 1975. Available on Chaitin's home page.

[9] G. Chaitin. Information theoretic characterizations of infinite strings. Theoret. Comput. Sci., 2:45-48, 1976. Available on Chaitin's home page.

[10] G. Chaitin. Algorithmic Information Theory. Cambridge University Press, 1987.

[11] G. Chaitin. Incompleteness theorems for random reals. Advances in Applied Math., pages 119-146, 1987. Available on Chaitin's home page.

[12] J.P. Delahaye. Information, complexité, hasard. Hermès, 1999 (2d edition).

[13] R. Downey and D. Hirschfeldt. Algorithmic randomness and complexity. Springer, 2006. To appear.

[14] W. Feller. Introduction to probability theory and its applications, volume 1. John Wiley, 1968 (3d edition).

[15] M. Ferbus and S. Grigorieff. Kolmogorov complexities $k_{\min }, k_{\max }$ on computable partially ordered sets. Theoret. Comput. Sci., 352:159-180, 2006 .

[16] M. Ferbus and S. Grigorieff. Kolmogorov complexity and set theoretical representations of integers. Math. Logic Quarterly, 52(4):381-409, 2006.

[17] P. Gács. Lectures notes on descriptional complexity and randomness. Boston University, pages 1-67, $1993 . \quad \mathrm{http}: / \mathrm{cs}-$ pub.bu.edu/faculty/gacs/Home.html.

[18] S. Grigorieff and J.Y. Marion. Kolmogorov complexity and nondeterminism. Theoret. Comput. Sci., 271:151-180, 2002.

[19] Y. Gurevich. The Logic in Computer Science Column: On Kolmogorov machines and related issues. Bull. EATCS, 35:71-82, 1988.

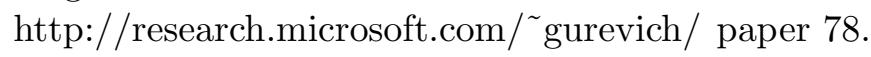

[20] D. Knuth. The Art of Computer Programming. Volume 2: seminumerical algorithms. Addison-Wesley, 1981 (2d edition). 
[21] A.N. Kolmogorov. Grundbegriffe der Wahscheinlichkeitsrechnung. Springer-Verlag, 1933. English translation 'Foundations of the Theory of Probability', Chelsea, 1956.

[22] A.N. Kolmogorov. On tables of random numbers. Sankhya, The Indian Journal of Statistics, ser. A, 25:369-376, 1963.

[23] A.N. Kolmogorov. Three approaches to the quantitative definition of information. Problems Inform. Transmission, 1(1):1-7, 1965.

[24] A.N. Kolmogorov. Some theorems about algorithmic entropy and algorithmic information. Uspekhi Mat. Nauk, 23(2):201, 1968. (in russian).

[25] A.N. Kolmogorov. Combinatorial foundation of information theory and the calculus of probability. Russian Math. Surveys, 38(4):29-40, 1983.

[26] A.N. Kolmogorov and V. Uspensky. Algorithms and randomness. SIAM J. Theory Probab. Appl., 32:389-412, 1987.

[27] A. Kučera and T.A. Slaman. Randomness and recursive enumerability. SIAM J. on Computing, 2001. to appear.

[28] L. Levin. On the notion of random sequence. Soviet Math. Dokl., 14(5):1413-1416, 1973.

[29] L. Levin. Random conservation inequalities; information and independence in mathematical theories. Information and Control, 61:15-37, 1984 .

[30] M. Li and P. Vitanyi. An introduction to Kolmogorov complexity and its applications. Springer, 1997 (2d edition).

[31] D. Loveland. A new interpretation of von Mises's concept of random sequence. Z. Math. Logik und Grundlagen Math., 12:279-294, 1966.

[32] D. Loveland. A variant of the Kolmogorov concept of complexity. Information and Control, 15:510-526, 1969.

[33] Michael Machtey and Paul Young. An introduction to the general theory of algorithms. North-Holland, New York, 1978.

[34] P. Martin-Löf. The definition of random sequences. Information and Control, 9:602-619, 1966. 
[35] P. Martin-Löf. Complexity of oscilations in infinite binary sequences. Z. Wahrscheinlichkeitstheorie verw. Geb., 19:225-230, 1971.

[36] J. Miller and L. Yu. On initial segment complexity and degrees of randomness. Trans. Amer. Math. Soc. to appear.

[37] W. Paul. Kolmogorov's complexity and lower bounds. In L. Budach, editor, Proc. 2nd Int. Conf. Fundamentals of Computation Theory, pages 325-334. Akademie Verlag, 1979.

[38] B. Russell. Mathematical logic as based on the theory of types. Amer. J. Math., 30:222-262, 1908. Reprinted in 'From Frege to Gdel A source book in mathematical logic, 1879-1931', J. van Heijenoort ed., p. 150$182,1967$.

[39] P. Schnorr. A unified approach to the definition of random sequences. Math. Systems Theory, 5:246-258, 1971.

[40] A. Shen. Kolmogorov complexity and its applications. Lecture Notes, Uppsala University, Sweden, pages 1-23, 2000. http://www.csd.uu.se/ vorobyov/ Courses/KC/2000/all.ps.

[41] A. Shen and V. Uspensky. Relations between varieties of Kolmogorov complexities. Mathematical systems theory, 29:271-292, 1996.

[42] R. Solomonoff. A formal theory of inductive inference, part 1. Information and control, 7:1-22, 1965.

[43] R.M. Solovay. Draft of paper (or series of papers) on chaitin's work, done for the most part during the period of sept.-dec. 1974. Unpublished manuscript, IBM Thomas Watson Research Center, Yorktown Heights, NY.

[44] R.M. Solovay. A model of set theory in which every set of reals is Lebesgue measurable. Annals of Mathematics, 92:1-56, 1970.

[45] R.M. Solovay. A version of $\Omega$ for which ZFC can not predict a single bit. Centre for Discrete Math and Comp. Sc., Auckland, New Zealand, 104:1-11, 1999. http://www.cs.auckland.ac.nz/staff-cgi$\mathrm{bin} / \mathrm{mjd} /$ secondcgi.pl.

[46] V.A Uspensky, A.L Semenov, and A.Kh Shen. Can an individual sequence of zeros and ones be random. Russian Math. Surveys, 41(1):121189, 1990. 
[47] J. Ville. Etude critique de la notion de Collectif. Gauthier-Villars, 1939.

[48] R. von Mises. Grundlagen der wahrscheinlichkeitsrechnung. Mathemat. Zeitsch., 5:52-99, 1919.

[49] R. von Mises. Probability, Statistics and Truth. Macmillan, 1939. Reprinted: Dover, 1981.

[50] A. Zvonkin and L. Levin. The complexity of finite objects and the development of the concepts of information and randomness by means of the theory of algorithms. Russian Math. Surveys, 6:83-124, 1970. 